\title{
Apuntes sobre la enseñanza filosófica en la Nueva Academia ${ }^{1}$
}

\section{Notes about philosophical teaching in the New Academy.}

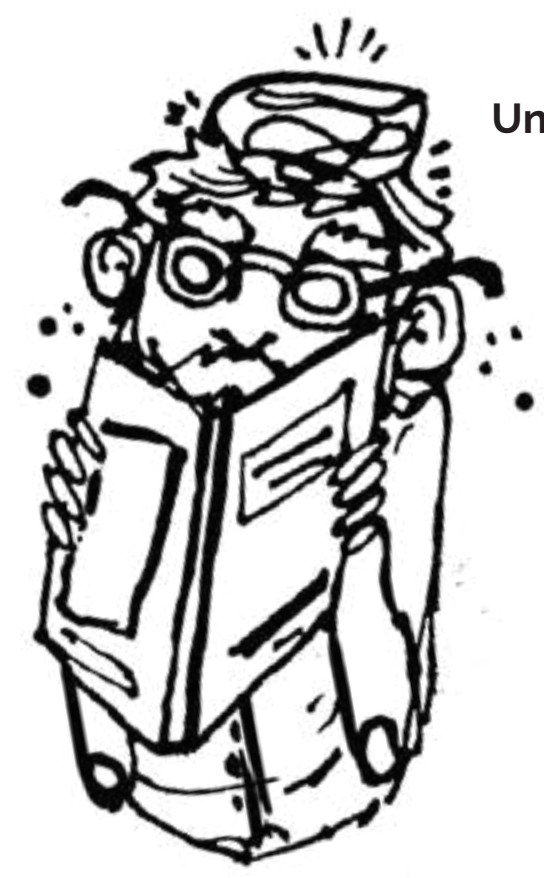

Marco Arturo Rojas Landaeta ${ }^{2}$ Universidad Experimental Simón Rodriguez, Venezuela

1 Las ideas delartículo constituyen esas reflexiones lindantes a todo trabajo de investigación académica, en este caso, la tesis intitulada "Filosofia y autoridad en Cicerón, del De oratore al De finibus"

2 Licenciado en Filosofía de la Universidad Central de Venezuela, por concluir el Magister de filosofía en la Universidad Simón Bolivar y el Doctorado el Filología Clásica de la Universidad Nacional de Educación a Distancia, España. Profesor de la cátedra de Filosofía, Educación y Pensamiento Latinoamericano en la Universidad Experimental Simón Rodriguez. E-mail: rojaslanda@hotmail.com 


\section{Resumen:}

Varios hechos concurren en el desconocimiento de una posible doctrina neoacadémica: a) su fundador, Carnéades, no escribió nada; b) las transcripciones de sus discursos, por parte de discipulos directos, se perdieron; y c) la visión de los neoacadémicos como simples disputadores preocupados exclusivamente por refutar. Sin embargo, el presente trabajo aspira entrever lo que habrian sido aspectos de una posible doctrina educativa en dicha escuela, a partir de la lectura de los diálogos ciceronianos. Con este propósito, se centrará la atención, en primer término, en el testimonio del Arpinate sobre el supuesto rechazo neoacadémico a que se anteponga la autoridad a la razón, y la consecuente defensa de la libertad de juicio en el aprendiz; en segundo término, en la implementación de los métodos dialécticos a los que se recurriria para superar la autoridad en la enseñanza; y, por último, en los posibles fundamentos ontológicos de tal propuesta pedagógica. La concordancia de estos elementos hará que se concluya la posible existencia de un pensamiento educativo sistemático en la nueva Academia.

Palabras clave: Nueva Academia, autoridad, enseñanza, Cicerón, métodos dialécticos

\section{Abstract:}

Several facts come together at the unknowledge of a possible neo-academic teaching: a) its founder, Carneades, wrote nothing; b) the transcriptions of his speeches done by his own disciples went lost; and c) the neo-academics' vision of simple contenders who are worried only with refuting. However, this work wants to provide a glimpse of the main aspects of what could be a possible educative doctrine in such a school, from the reading of the ciceronians dialogues. Taking into account this goal, we will focus, at first moment, in the testimony of Apricate concerning the supposed neo-academical rejection of the idea that reason comes before authority. The consequent defense of the freedom of judgement of the apprentice. As a second moment, in the implementation of dialectic methods in which they would support to overcome the authority at teaching. Finally, in the possible ontological foundations of such pedagogical proposal. The concordance of those elements will conclude about the possible existence of a systematic educative thought in the new academy.

Keywords: New Academy, authority, teaching, Cicero, dialectical methods 


\section{Notes sur l'enseignement philosophique dans la nouvelle Académie}

\section{Résumé :}

Plusieurs faits concordent avec l'ignorance d'une possible doctrine néoacadémique ; A) son fondateur, Carnéades ; n'en a rien écrit. B) les transcriptions de ses discours, faites par de disciples directs ; sont perdues. C) la vision des néoacadémiques, comme des simples disputeurs inquiets sans autre but que ce de réfuter. Cependant, le travail à présenter a été fait en souhaitant apercevoir quels seraient les aspects d'une possible doctrine éducative y appliquée, à partir de la lecture des dialogues cicéroniens. Avec cet intention-là, l'attention sera mise, d'abbord, dans le témoignage de l'Arpinat sur le prétendu rejet néo-académique à celui-là où l'autorité est en avant de la raison, et la défense conséquent de la liberté de jugement de l'apprenant ; ensuite, dans l'implémentation des méthodes dialectiques lesquelles pourraient être abordées afin de surmonter l'autorité dans l'enseignement, et finalement, dans les possibles fondements ontologiques de celle proposition pédagogique-là.

Mots-clés: Nouvelle Académie, autorité, enseignement, Cicéron, méthodes dialectiques 


\section{Notas sobre o ensino filosófico na nova Academia}

\section{Resumo:}

Vários fatos têm desconhecimento de uma possivel doutrina neoacadêmica: a) seu fundador, Carnéades, não escreveu nada; b) as transcrições dos discursos, por parte de seus discípulos diretos, se perderam; e c) a visão dos neoacadêmicos como simples disputadores preocupados exclusivamente por refutar. Embora, o presente artigo deixa entrever o que teriam sido aspetos de uma possivel doutrina educacional nessa escola, partindo da leitura dos diálogos ciceronianos. Com este propósito, centra-se a atenção, em primeiro lugar, no testemunho do Arpinate sobre a suposta rejeição neoacadêmica acima a autoridade à razão, e consequentemente, a defensa da liberdade do julgamento no aprendiz; no segundo momento, na implementação dos métodos dialéticos no percurso de ultrapassar a autoridade no ensino; e, por último, nos possiveis fundamentos ontológicos dessa proposta pedagógica. A concordância desses elementos fara que, conclua-se a possivel existência de um pensamento educativo sistemático na nova Academia.

Palavras-chave: Nova Academia, autoridade, ensino, Cícero, métodos dialéticos. 


\section{Introducción}

En los últimos estudios sobre la educación en el periodo helenístico, predomina el interés por distintos aspectos de la enseñanza literaria (v. gr., Morgan, 1998; Bowman y Woolf, Eds., 1994) y, en menor medida, de la instrucción retórica (v. gr., Pernot, 1993)1. Pero, hasta donde se ha podido constatar, solo el excelente trabajo de Elizabeth Asmis (2001), sobre la educación epicúrea, se preocupa por la enseñanza dentro de círculos pedagógicos distintos a la instrucción general, como fue el caso de las escuelas filosóficas.

Por lo anterior, el presente artículo pretende adelantar reflexiones que posibiliten el bosquejo de lo que habría sido la educación en una de las escuelas filosóficas más importantes de la época: la nueva Academia. Para cumplir con este esfuerzo exploratorio, se estudiará, en primer lugar, la relación entre autoridad y libertad de juicio en la mencionada escuela, al tratar de desarrollar el problema desde la perspectiva del aprendiz y del maestro. En segundo lugar, se abordarán los métodos con los cuales se pretendia evitar la influencia de la autoridad: el in utramque partem (argumentación en pro y en contra), el omnes res audire (conocer todas las doctrinas) y el contra omnia dicere (refutar todo lo dicho). Y, por último, se buscará el posible fundamento ontológico del rechazo de la autoridad en la enseñanza filosófica.

Toda afirmación sobre la doctrina neoacadémica necesariamente comporta un grado de vaguedad. Para empezar, su iniciador, Carnéades, al asumirse seguidor de Sócrates, no escribió nada. Se piensa que algunos discipulos directos -en particular, Clitómaco- habrian transcrito sus discursos (Schofield, 2002, n. 5, p. 323); sin embargo, los susodichos se perdieron. Quedan escritas las opiniones de lectores de los discípulos de Carnéades, esto es, quedan fuentes de tercera mano (Schofield, 2002, p. 323). Las principales, según Schofield (2002, n. 1, p. 323), las Academicae de Cicerón y Adversus mathematicos de Sexto Empírico. Para cumplir con los objetivos del presente trabajo exploratorio, nos limitaremos a una lectura de la obra dialogada ciceroniana.

1 Para el estudio de la educación antigua, continúa siendo referencia obligada la obra de Marrou (1948). Por su parte, la obra de Bonner (2012) es fundamental para la comprensión de la educación romana durante la época del helenismo. El trabajo de Too (2001) ofrece un análisis más reciente de la educación en el mundo greco-romano. 


\section{Autoridad y libertad de juicio}

El rechazo que el Arpinate establece entre autoridad y filosofía en su obra es el fundamento al cual recurren estudiosos (Fox, 2007; Atkins, 2013) con el fin de oponerse a novedosas investigaciones retóricas (Dugan, 2005; Van der Blom, 2010; Culpepper, 2010) que pretenden convertir los escritos del autor en un mero esfuerzo persuasivo para promover su prestigio. Sin embargo, no se ha prestado suficiente atención a las implicaciones educativas de tal rechazo a la autoridad. En el De natura deorum I 10, Cicerón, al reflexionar sobre las circunstancias en las que ha escrito su obra, así como sus consecuencias, realiza una súbita advertencia: "Quienes se preguntan, por su parte, qué es lo que opinamos personalmente sobre cada asunto, lo hacen con mayor curiosidad de la necesaria, porque, cuando se discute, no ha de buscarse tanto el peso de la autoridad como el de la razón" (Trad. Ángel Escobar, 1999a)

Los diálogos ciceronianos están diseñados de tal modo que no se puede saber con certeza cuál es la posición definitiva del autor. La principal razón del hecho se encuentra en su estructura dialéctica, la cual se desarrolla a través de dos largos discursos, de igual peso argumentativo, que se oponen entre sí. Un buen ejemplo seria el De divinatione, pues en el primer libro se encuentra un amplio discurso sobre la eficacia adivinatoria romana, pero en el segundo libro hay otro amplio discurso refutando esa práctica (Schofield, 1986, p. 56).

¿Cuál es la verdadera opinión del autor sobre el tema? Si nos atenemos a la advertencia del De natura deorum I 10, esa pregunta es precisamente la que se debe evitar. Sin embargo, desde la antigüedad los intérpretes han mostrado el exceso de curiosidad que Cicerón critica, alesforzarse en desentrañarelverdadero pensamiento del autor al respecto (Schofield, 1986, p. 56). ¿Tal curiosidad no desplaza el centro de atención del objetivo que el autor se proponía? En todo caso, para Cicerón esa curiosidad termina anteponiendo la autoridad (auctoritas) a la razón (ratio) en la disputa filosófica.

El prevalecer de la auctoritas trae otra consecuencia. Inmediatamente después de las líneas citadas, Cicerón prosigue: "es más la autoridad de los que profesan la 
enseñanza incluso constituye un obstáculo, la mayoría de las veces, para quienes quieren aprender, porque estos dejan de aplicar su propio juicio y dan por válido lo que ven que ha dictaminado aquel a que aprueban" (I 10). Las palabras muestran lo que debió ser uno de los objetivos principales de la nueva Academia -o, al menos, de la Academia de Cicerón- esto es, que el aprendiz ejerza su propio juicio (suum iudicium adhibere).

Apoyándose en los anteriores fragmentos, Carlos Lévy (1992, p. 121) considera la necesidad de concebir al neoacadémico -y, agregamos nosotros, al iniciado de esa escuela- como un juez pronunciando su juicio sobre los argumentos presentados. Es por ello que Lévy también concluye que en la nueva Academia debió existir una "une totale liberté d'esprit" (1992, p. 121). En consecuencia, el "aplicar su propio juicio" de la enseñanza neoacadémica supone una absoluta libertad de pensamiento en el aprendiz. Sin embargo, tal libertad no se alcanzaría si el juicio del iniciado es sustituido por el juicio del sabio. Hecho que acontece cuando el aprendiz da por válido el dictamen (la auctoritas) del maestro simplemente por el reconocimiento que todos dan a su sabiduria.

La presencia de este problema en la educación filosófica antigua puede parecer extraña si prevalece la imagen de Sócrates disputando entre los pórticos, pero Cicerón testifica su existencia. Asi continúa diciendo en I 10:

$Y$ por cierto que no suelo aprobar lo que tenemos entendido acerca de los pitagóricos, de quienes cuentan que, si afirmaban algo durante el desarrollo de una discusión y entonces se les preguntaba por qué era así, solian responder que 'él en persona lo dijo' ('él en persona', por lo demás, era Pitágoras): una creencia preestablecida tenía tanto poder que incluso sin razonamiento alguno prevalecía su autoridad (Trad. Ángel Escobar, 1999a).

Es una incógnita que escapa a los alcances del presente trabajo determinar hasta qué punto esta enseñanza era común a otras escuelas anteriores y contemporáneas al Arpinate. Si nos atenemos a la preocupación que muestra el autor, se trataría de una práctica difundida. Aquello que sí parece claro es que Cicerón, bajo la influencia de la nueva Academia, se opone a una enseñanza de este tipo. 
Es interesante observar que no se cuestiona el contenido de verdad de lo afirmado por Pitágoras. Sus afirmaciones pudieron estar fundadas en sólidas razones. Pero esta no es la ratio que preocupa a la nueva Academia en los fragmentos analizados. La ratio que se defiende en ellos es la que posibilita juzgar libremente afirmaciones de reconocidos sabios. Medio que evitaría sustituir el propio juicio por el de la autoridad, aunque esta autoridad diga la verdad. Tal preocupación nos hace pensar en la enseñanza actual; particularmente, la universitaria, en la cual se puede observar al estudiante aceptar como verdaderas -y pueden serlo- las afirmaciones de un investigador por el simple hecho de su prestigio, pero dejando con ello de aplicar su propio juicio (suum iudicium adhibere).

En el prólogo al Lucullus, Cicerón asevera que los neoacadémicos son más libres e independientes (liberiores et solutiores), pues, por un lado, mantienen integra su potestad de juzgar (Integra nobis est iudicandi potestas) y, por el otro, no están obligados a defender doctrinas prescritas e impuestas por algunos (8-9). De nuevo, encontramos el testimonio de que en la enseñanza filosófica de la época existía más imposición y autoridad que razón (ratio). Y el origen de esto, continúa diciendo el autor, se encuentra en que los seguidores de otras escuelas filosóficas:

...primero, están ya coaccionados antes de poder juzgar qué es lo mejor; después, en la etapa más frágil de su edad, o influidos por algún amigo, o cautivados por un solo discurso de alguien a quien oyeron por primera vez, juzgan sobre cosas desconocidas y, cualquiera que sea el sistema hacia el cual fueron arrastrados como por una tempestad, se aferran a él como a una roca (Trad. Julio Pimentel, 1990).

La imposición doctrinal que se denuncia tendría su explicación en causas de tipo educativo: los iniciados no pueden juzgar adecuadamente debido a algún género de violencia o porque, al ser muy jóvenes todavía, juzgan bajo el influjo del amigo o del primer sabio que escuchan. Un rápido análisis de estas causas podria fácilmente concluir que la verdadera fuente del problema es solo la autoridad: ella es la que coacciona el juicio o lo precipita. La prontitud de la conclusión quizá se deba a la introducción de una premisa de nuestra época, y no de la del Arpinate. 
Los enfoques educativos más innovadores del presente asumen la autoridad como uno de sus principales enemigos. ¿Cómo leer, entonces, esas líneas del Lucullus y no adjudicarle toda la responsabilidad a la auctoritas? Sin embargo, el problema no parece tan simple. La pregunta que cabria formular es a quiénes atribuye el autor la imposición doctrinal en su tiempo: ¿a los que descuidan el ejercicio de su libertad de juicio o a los que imponen su autoridad? El asunto no es claro en el fragmento, pero la imagen final parece hacer también responsables a los que descuidan su libertad de juicio, de ahí la ironía con que son representados aferrados a la roca (entiéndase doctrina) en medio de la tempestad. Esto parece corroborarse en las críticas que inmediatamente se les dirigen:

Pues eso que dicen: que ellos creen totalmente a aquel que juzgan haber sido sabio, yo lo aprobaría si esto mismo lo hubieran podido juzgar los inexpertos e indoctos... pero, suponiendo que lo hayan podido, lo pudieron después de haber oído todas las doctrinas y conocido las sentencias de los demás; en cambio, emitieron su juicio después de haber oído una sola vez un sistema, y se refugiaron en la autoridad de uno solo (1990, 9-10).

Aqui no se cuestiona al sabio por imponer su juicio con el respaldo del prestigio, sino más bien el "facilismo" con que el iniciado entrega su asentimiento a la primera doctrina escuchada. Estas líneas permiten percibir que es más cómodo seguir el dictamen de una sola persona que buscar el juicio propio.

Semejante economía inherente al juzgar del aprendiz nos trae de nuevo a la educación actual, pues no es compatible con la percepción que existe de la dinámica del aula de clase. Preocupada por erradicar la autoridad, la pedagogía dominante convierte al docente en el único responsable de la imposición doctrinal a un auditorio de estudiantes deseosos de libertad de juicio. Si lo que creemos ver en Cicerón es cierto, esta perspectiva no es del todo precisa, ya que es mucho menos fatigoso y práctico seguir la opinión de un solo especialista que juzgar acerca de los múltiples puntos de vista sobre un tema. Con lo cual, la aparición de la autoridad en el aula de clase no es responsabilidad exclusiva del profesor, sino también del sentido práctico del estudiante. 
Este fenómeno sorprende tanto a Cicerón -y quizá a la nueva Academia- que termina diciendo: "Pero no sé por qué motivo la mayoría prefiere errar y defender con muchísima aspereza la doctrina que ama, a investigar sin pertinacia qué es lo que se dice en la forma más consistente" $(1990,9-10)^{2}$

\section{Libertad de juicio y métodos dialécticos}

Según Schofield (2008, p. 71), el rechazo ciceroniano a la autoridad va unido a un método dialéctico. El estudioso remite al De divinatione II 150:

... siendo propio de la Academia el no imponer ningún juicio propio, aprobar aquello que parece asemejarse más a lo verdadero, comparar unas causas con otras y exponer lo que puede decirse en contra de cada opinión, asi como dejar intacto y libre el juicio de los oyentes, sin ejercer sobre él autoridad alguna, mantendremos esta costumbre (Trad. Ángel Escobar, 1999b).

Se reitera lo visto, la nueva Academia deja intacto (intenger) y libre (liber) el juicio del aprendiz, esto es, no impone su autoridad (auctoritas).

Sin embargo, tal como resalta Schofield, en el fragmento parece unirse un método dialéctico al respeto de la libertad de juicio. Para precisar la fisonomía de esta dialéctica, Schofield (1986, p. 63) centra su atención en las palabras "comparar causas" (conferre causas), y opina que poseen el mismo significado con que, en la introducción del De divinatione, se habla de comparar argumentos con argumentos (7-8: argumenta cum argumenta comparamus). Comparar causas es comparar argumentos y, como estas últimas palabras se encuentran en la introducción de la obra, es de suponerse que manifiestan el método expositivo seguido en el escrito. Ya se constató al comienzo de nuestro artículo: en el primer libro del De divinatione se argumenta en favor de la adivinatoria romana; en el segundo, en contra (supra, p.2). El diálogo compara argumentos.

En el De fato 1, Cicerón confiesa que ciertas circunstancias le impidieron escribir esta obra del mismo modo que el De natura deorum y el De divinatione, en las cuales:

2 Es interesante observar cómo para Cicerón puede existir falta de voluntad al juzgar sobre las diversas opiniones de un tema, pero no para defender vehementemente la única opinión que se escuchó. 
"el discurso se desarrollaba... sin interrupción, con argumentos en pro y en contra (in utramque partem), para que cada uno pudiese probar con mayor facilidad aquello que a cada uno le parecía más probable" (Trad. Ángel Escobar, 1999b). Se corrobora lo dicho por Schofield: el De divinatione fue diseñado para comparar argumentos. En el fragmento, asimismo, se indica el nombre por el cual será conocido posteriormente dicho método: el in utramque partem. Además, se refieren dos de sus características: a) consta de dos discursos continuos (orationes perpetuas); b) los discursos se oponen entre sí. Pero lo que debe resaltarse aqui es que la nueva Academia buscaria mantener la potestad de juicio del aprendiz, a través de tal dialéctica.

No hay que confundir esta dialéctica con otra de gran influencia educativa, la, a veces llamada, mayéutica socrática3. Sócrates interrogaba a su oponente o aprendiz, por medio de preguntas cortas para respuestas breves. Dicha mecánica permite, entre otras cosas, una constante interacción entre el participante y el maestro. En el in utramque partem, el aprendiz debe escuchar dos discursos continuos sin interrumpir y dar su juicio solo al final del debate (infra, p. 9). Se tiene la sensación propia de la distancia de un espectáculo o la de un típico salón de clase.

En el libro I del De oratore, se encuentra otro excelente ejemplo del in utramque partem, que permite ahondar en su naturaleza y aplicación. El libro contiene varias sesiones de confrontación de argumentos a las que asisten dos jóvenes (Sulpicio y Cota) con la intención de aprender. En la primera sesión, Craso alaba la capacidad de la elocuencia para organizar al hombre en sociedad. Este discurso es refutado de inmediato por otro de Escévola (30). Craso replica y Marco Antonio sale en ayuda de Escévola con otro discurso (80-95).

Aqui el lector comienza a percibir algo extraño, pues los cuatro discursos poseen el mismo peso argumentativo. ¿Cuáles, entonces, la opinión del autor? Ya vimos que esa curiosidad debe evitarse. El libro termina con dos largos discursos que igualmente se contraponen: Craso diserta sobre aquellas cosas más útiles que la técnica para el orador, Marco Antonio lo refuta punto por punto (166-262). La formulación correcta de la pregunta parecería ser la siguiente: ¿cuál de las dos opiniones es más cierta? Así, al finalizar el último discurso del libro I del De oratore, Cicerón dice: "Habiendo dicho Antonio estas cosas, claramente se veía que Sulpicio y Cota dudaban acerca de cuál de los dos discursos parecía acercarse más a la verdad"4.

3 Aunque, según Cicerón, tal método se inspira en la dialéctica socrática (De divinatione II 150).

4 La traducción es nuestra. 
El fragmento muestra lo que debió ser uno de los comportamientos que la nueva Academia aspiraba a provocar en el aprendiz. Si esto es correcto, el fragmento también muestra otra característica esencial de la educación de dicha escuela: el dudar (dubitare) como condición previa para que el iniciado emita su juicio con entera libertad. Circunstancia que platea la pregunta: ¿la duda realmente posibilita el juicio? A simple vista, pareciera que sí, aunque el problema no es nada obvio.

Según el testimonio de Cicerón, la imposibilidad de saber cuál juicio es verdadero - falso condujo a Arcesilao a proponer la suspensión del juicio, la conocida epojé (Academicae II 58-59). En caso de ser exacta la propuesta de Arcesilao, se podría afirmar que la duda es un medio erróneo para estimular en el aprendiz su potestad de juicio, pues conduce al escepticismo. Resulta paradójico, pero la duda que propiciaria la imposibilidad del juicio en la Academia iniciada por Arcesilao y Carnéades ${ }^{5}$ es la que posibilita el juicio libre en el academicismo de Cicerón.

La educación actual, sin embargo, estaría de acuerdo con nuestro autor. Dada su inclinación por rechazar verdades inamovibles en el salón de clase, se podría calificar de escéptica a la educación de hoy en día. Ahora bien, tanto dudar, tanto relativismo, ¿no conducirá al estudiante a ser indiferente por juicio que constantemente se aspira de él? Si se puede dudar de todo conocimiento, ¿no será el comportamiento más natural el no pronunciarse ante nada? Pero se tiene la sensación de que la pedagogía actual asume una postura completamente opuesta. En el presente, la mayoría del profesorado afirmaría que la construcción del saber, en tanto que acto de creación libre, parte de la erradicación de verdades inmutables dentro de la institución educativa. Eliminado el dogmatismo, los alumnos se entregarían con ánimo a la indagación.

5 Aunque, como es sabido, Arcesilao propuso la posibilidad del juicio "razonable" en la vida práctica (Sexto Empírico, Adversus mathematicos, VII 158). Carnéades habria mantenido, con ciertas modificaciones, la misma postura. Sin embargo, desde la antigüedad, hay quienes han afirmado que Carnéades igualmente planteó la posibilidad de emitir juicios sobre las cosas, aunque sabiendo que eran meras opiniones, pues no existe ninguna certidumbre (Lucullus 67). Esta orientación del pensamiento de Carnéades podría explicar cómo el escepticismo neoacadémico de la época de Cicerón pediría juicios teóricos a sus aprendices. No obstante, parece que Cicerón no estaba de acuerdo con esta orientación del pensamiento de Carnéades (Lucullus 108). A lo que se debe agregar que el Arpinate fue discipulo de Filón de Larisa, quien, según investigaciones actuales (Brittain, 2001), habría introducido la Academia en una suerte de falibilismo: pueden existir verdades, aunque sujetas al error. Un marco que permitiria el pronunciamiento de juicios. El problema acerca de la naturaleza del juicio promovido en la enseñanza académica ciceroniana queda abierto, pero si debe quedar claro que, contrario a lo que ocurre en Arcesilao, después del dubitare producto del in utramque partem no viene la epojé, sino otro juicio, cuya principal caracteristica es su libertad. 
El Arpinate -y la nueva Academia que representa- apuesta por el mismo esfuerzo investigativo, pero, según creemos ver, no pareciera estar muy seguro de que la incertidumbre escéptica lo favorezca. Dentro de aquellas palabras del Lucullus, en la cuales se cuestionaría al aprendiz no cuidar su libertad de juicio (supra, p. 4), el autor defiende a su escuela de las consecuencias del escepticismo, al aseverar que los neoacadémicos también buscan la verdad, aunque libre de toda controversia (7-8).

Sin embargo, para cumplir con esta exigencia, continúa diciendo Cicerón, hay que sortear muchas dificultades, tanto en la cosa misma como en la debilidad de los juicios. $Y$ sabiendo lo que semejante estado de incertidumbre ocasiona, advierte que no hay que dejarse vencer por la fatiga (defatigatio), pues provocaría el desmayo y el abandono en la entrega investigativa (7-8). Para el autor, de la erradicación del dogmatismo, no se sigue un aumento del esfuerzo indagatorio por la verdad. $\mathrm{Y}$, como estas afirmaciones se dicen dentro de la crítica al manejo del juicio por parte del aprendiz, es de esperarse que la defatigatio también afecte a los iniciados.

Es más, se puede aventurar, a partir del fragmento del Lucullus, que los neoacadémicos eran conscientes de que su lucha contra el dogmatismo podia traer consecuencias adversas en la enseñanza, pues, tal como entendian la iudicandi potestas, implicaba abarcar todo lo dicho acerca de un tema, lo cual, además de ocasionar defatigatio, encaminaba al iniciado a preferir aferrarse a cualquier doctrina como a una piedra en medio de la tempestad (supra, p. 4), es decir, a practicar un dogmatismo superficial. Basándonos en esto, tendría algún sentido afirmar que, para la nueva Academia, el espíritu práctico del aprendiz lo inclina naturalmente tanto a la autoridad como al dogmatismo: dos caras de una misma moneda.

Sin embargo, a pesar de lo que se acaba de sugerir, el neoacademicismo auspicia un esfuerzo investigativo que tendría como punto de partida el dubitare. Pero, ¿cómo superar el "quietismo" indagatorio al que, según el temor ciceroniano, podría encaminar la incertidumbre escéptica? En el De oratore, los jóvenes Sulpicio y Cota, después de escuchar el in utramque partem, no preguntan el parecer de los que hablan, se limitan, sin que se les pida, a reflexionar cuál de los dos discursos se aproxima más a la verdad. Es evidente que conocen el método dialéctico de la nueva Academia, pues lo más natural es interrogarse por la opinión real del autor. 
Pero hay otros diálogos en los cuales, al concluirel in utramque partem, explícitamente se pide el juicio a los que escuchan. Así, casi al final de la Academica priora, Cicerón evita cortésmente las pretensiones de Lúculo de seguir disputando, se vuelve hacia los oyentes -signo manifiesto de que esa es su finalidad-y les pide su juicio sobre lo discutido (148). En las últimas líneas del libro Il del De finibus, ocurre algo semejante: ante la posible réplica del epicúreo Torcuato, Cicerón interrumpe nuevamente la disputa diciendo que lo más justo es pedirle la opinión al joven Triario sobre lo debatido (119).

En estos casos, el sentido del in utramque partem no parece estar claro para los participantes de la disputa, por lo que el autor debe detenerla y retomar el objetivo original: que el oyente juzgue sobre el contenido de verdad de los discursos opuestos. Plasmar en el diálogo la preocupación de rectificar el curso de la discusión, sin duda, muestra una estrategia para que el lector asuma precisamente ese sentido; pero también manifiesta lo que debió ser la práctica neoacadémica al usar el in utramque partem frente al aprendiz: deliberadamente pedirle su juicio. Quizá sea esta sencilla pregunta ${ }^{6}$ una de las formas con las cuales la nueva Academia de Cicerón buscaba superar la epojé de Arcesilao, suprimir la defatigatio en la enseñanza y, por último, garantizar la libertad de juicio. Una pregunta no anterior a la duda, sino posterior.

Tal vez, la nueva Academia muestre preocupación por la defatigatio a la que conduciria el in utramque partem, mas no por la eficacia de este método para anular la autoridad del maestro. Y parece tener sentido, pues si el aprendiz escucha el discurso de un afamado sabio, tenderá a asumir tales palabras como el verdadero juicio del que habla; pero si al día siguiente escucha del reconocido sabio otro discurso tan persuasivo como el primero, aunque en sentido opuesto, ¿cuál de los dos discursos puede ser respaldado con la autoridad del maestro?

Semejante perspectiva invita a reflexionar sobre cierta peculiaridad del in utramque partem ciceroniano y sus consecuencias educativas. Es conocida la importancia que poseía la jerarquía entre el romano antiguo (Adcock, 2000, pp.13-14; Brunt, 1988, p. 90). Esta se alcanzaba a través de la dignitas: el reconocimiento público de los éxitos políticos y militares en beneficio del Estado (Adcock, 2000, p. 13; Gruen, 1995, p.

6 Resulta sencilla, pues - tal como se encuentra formulada en los diálogos- parece carecer de la complejidad mayéutica de las preguntas socráticas (Bravo, 1990, p. 85). 
47). Por el respeto a su dignitas, el jerarca romano habría dado su vida7. Ahora bien, la auctoritas es la voz, la opinión de la dignitas (Balsdon, 1960, p. 45). De ahí que si se respetaba rigurosamente la dignitas de un romano, en la misma proporción se respetaban sus opiniones, esto es, su auctoritas. El ciudadano de menor rango, o que careciera de él, se contentaba con seguir disciplinadamente las palabras del de mayor rango.

Esta arraigada mecánica social, como es de suponer, se reflejaba en la educación tradicional del joven romano: el tirocinium fori. Según Bonner (2012, pp. 84-85), el adolescente, al tomar la toga virilis, signo de su adultez, era confiado a un romano de la máxima dignitas posible, el cual debía ser seguido a todas partes por el joven. La búsqueda de esta figura de autoridad era ardua para la familia del adolescente, pero era llevada a cabo con meticulosidad. Tal esfuerzo muestra la importancia de la auctoritas en la educación romana. Conseguido el mejor princeps civitatis (De oratore I 105-106) al alcance, el noble adolescente debía seguirlo al foro, a los tribunales, a las asambleas públicas con el fin de imitarlo.

Pues bien, todos los personajes a los que recurre Cicerón en sus diálogos, para desplegar el in utramque partem, son exitosos hombres de Estado que han alcanzado los más altos puestos. En consecuencia, poseen la mayor dignitas posible y la auctoritas equivalente. Semejante estrategia al momento de redactar la obra no es de extrañar, si Cicerón pretende alguna influencia educativa en medio de una sociedad altamente conservadora. Además, Cicerón era defensor de la mos maiorum (la costumbre de los antiguos), que constituía un criterio de acción incuestionable entre los romanos ${ }^{8}$. Es explicable, entonces, que sus personajes encarnaran a aquellos ciudadanos que cualquier familia distinguida desearia para la educación de sus hijos.

7 Julio César afirmaba en su Bellum civile: "Sibi semper primam... fuisse dignitatem uitaque potiorem..." (I g) [Para él siempre fue más importante... la dignidad y más preferible que la vida]. Cicerón opinaba lo mismo en el Pro Sestio: "cum omnia semper ad dignitatem rettulissem nec sine ea quicquam expetendum esse homini in uita putassem..." (48) Itodo cuanto he hecho ha sido en vista a la dignidad, ni he pensado que el hombre deba esperar algo en la vida sin tal reconocimiento]. Para un investigación clásica de la obsesión romana de la época por la dignitas, cfr. Gruen (1995) y Balsdon (1960, p. 45).

8 Para un análisis actualizado de la mos maiorum en este sentido, cfr. la introducción de Van der Blom (2010). Introducción que está en dividida en Mos, maiores, and historical exempla in Roman culture and society y Mos, maiores, and historical exempla in Cicero. 
El problema es evidente: ¿cómo conjugar esta defensa de la autoridad con una escuela filosófica que rechaza precisamente su uso en la enseñanza?9 La solución a la dificultad ya ha sido esbozada: el in utramque partem neoacadémico. Cicerón un consularis de la máxima dignitas y auctoritas ${ }^{-10}$ desarrolla su enseñanza a través de dos discursos enfrentados -puestos en boca de otros tantos consulares- cuya paridad persuasiva harian imposible que el aprendiz asocie alguno de ellos con su autoridad.

Si nos atenemos al in utramque partem ciceroniano, tal método parecería pensado para mantener la figura de autoridad, al mismo tiempo que impediria la influencia de esta en el juicio del aprendiz. El esfuerzo del autor, por presentar solamente hombres de la más alta auctoritas frente a los oyentes y los lectores, muestra la asunción de que este es el camino correcto en la educación: el iniciado siempre debe escuchar al que posee la mayor autoridad. Sin embargo, las palabras de la autoridad deben ser juzgadas con entera libertad de juicio en la nueva Academia. Solución al problema: el in utramque partem.

Contrasta semejante salida con la que, en nuestra opinión, toma cada vez más fuerza en la educación actual. Ante la percepción de que la autoridad representa un grave obstáculo en todos los quehaceres -cosa absurda para un romano- se busca hoy en dia erradicarla en el maestro mismo, al eliminar en lo posible cualquier jerarquía entre él y los estudiantes. Un neoacadémico quizá habría dicho que eso es innecesario, ya que puede mantenerse la libertad de juicio del alumno, sin que por ello disminuya el respeto y la jerarquia del profesor.

9 Dos cosas muy distintas eran la enseñanza filosófica neoacadémica y la enseñanza tradicional romana. Sin duda, podian conjugarse para Cicerón, pero no para la mayoría de la aristocracia romana. La razón se encuentra en la aversión latina por la filosofía. Un hecho histórico puede servir de ilustración: en el 155 a. C. llegó a Roma la famosa embajada ateniense de los filósofos Carnéades, Diógenes y Filolao. Durante su estancia, Carnéades pronunció un persuasivo discurso en favor de la justicia y al dia siguiente otro, tan persuasivo como el primero, pero en sentido contrario. Como se observa, es el in utramque partem del que se ha venido hablando. Luego de esto, Catón el censor, liderando la aristocracia conservadora, expulsó a los filósofos de Roma mediante un senatusconsultum. Según Plutarco, la medida se habría tomado contra ellos: "... para que..., volviendo a sus escuelas, tratasen con niños griegos, y los jóvenes romanos escucharan, como antes, las leyes y los magistrados" (trad. Juan Guzmán, Vidas paralelas IV, Catón). El senatusconsultum buscaba proteger el tirocinium fori contra las innovaciones filosóficas. Para una exhaustiva investigación sobre el rechazo romano a la filosofía, cfr. Baraz (2012).

10 Cuando un romano alcanzaba la más alta magistratura del cursus honorum, el consulado, obtenía la designación de consularis para el resto de su vida. 
En el fragmento del Lucullus en el que se pretendió ver una crítica al iniciado por preferir la autoridad de un solo maestro (supra, p. 5), se puede plantear la presencia de otro método dialéctico neoacadémico en relación con la enseñanza. Según Cicerón, el aprendiz suele afirmar que alguien es sabio sin haber escuchado previamente todas las doctrinas (omnes res audire), con lo cual queda atrapado en la autoridad de uno solo. Por consiguiente, el omnes res audire parece constituir otro método para evitar que el prestigio del sabio influya en la potestad de juicio durante la instrucción filosófica.

La idea que podría encontrarse detrás de esto es que el esfuerzo de comparar las múltiples afirmaciones sobre un tema, además de permitir mayor aproximación a la verdad, acrecienta la distancia con respecto a los que formularon las opiniones. $\mathrm{Si}$, por ejemplo, un joven estudiante tiene el trabajo de confrontar todo lo dicho por sus maestros acerca de la justicia, se podría suponer que tal ejercicio desplazaría su atención del problemático prestigio de los docentes.

Sin embargo, los análisis previos mostraron dos preocupaciones ciceronianas que, por lo menos, manifiestan los peligros del omnes res audire. La primera, que el intento de abarcar todo lo dicho puede ocasionar defatigatio investigativa (supra, p. 9), lo que, a su vez, suscita la preferencia del espíritu práctico del iniciado por la opinión de un solo maestro. La segunda, que dicho iniciado tiende a juzgar sobre cuestiones desconocidas (supra, p. 4), bien sea a causa de su juventud o de lo influenciable de su juicio en presencia del amigo o del sabio. De ahí el peligro que significaría para su educación que escuche directamente una doctrina de boca del prestigioso filósofo que la propuso.

¿De qué modo, entonces, debe el aprendiz abarcar todo lo dicho sobre un tema? Líneas después de afirmar que no hay que anteponer la auctoritas a la ratio en el De natura deorum I 10, Cicerón vincula la aspiración del conocimiento de todas las cosas con un método que podría guardar relación con el omnes res audire. Él testimonia la existencia de un procedimiento "que consiste en poder disertar frente a todo (contra omnia dissere) y en no juzgar sobre asunto alguno sin reserva" (11-12). Inmediatamente, añade que tal método era el utilizado por Sócrates, Arcesilao y Carnéades, y que debía retomarse, "pues, si resulta grandioso poder comprender cada escuela por separado, icuánto mayor resulta comprenderlas todas!" (11-12). Obsérvese la importancia que parece poseer el conocimiento omniabarcante en la 
neoacademia ciceroniana, y cómo tan grandioso objetivo se lograría refutando cada uno de los sistemas.

Ahora bien, dado el valor que parece otorgársele aqui al conocimiento omnium rerum, se podría aventurar que el estudio exigido al aprendiz en Lucullus 9-10 es de naturaleza similar. Pero si esto es cierto, igualmente se podría suponer que el aprendiz debe oír (audire) a cada uno de los llamados sabios con una actitud refutativa. No se buscaria, entonces, que el iniciado acumule la opinión de todos los sistemas, tampoco que evalúe la proximidad a la verdad de las distintas propuestas (como quizá ocurriria en el in utramque partem), sino que intente refutar, al modo socrático, todo lo afirmado por los prestigiosos maestros.

¿El contra omnia dicere de Sócrates permite superar la autoridad en la enseñanza? Nada pareciera más apropiado. El rechazo a la autoridad es lo que se percibe cuando vemos a Sócrates refutando a aquellos afamados maestros de la virtud, los sofistas. La refutación socrática busca la ratio, no la auctoritas, tal como pretende igualmente la nueva Academia (supra, p. 2). En consecuencia, parece sostenible afirmar que el omnes res audire del que se habla en el Lucullus permite al iniciado enfrentarse solo a la reconocida opinión de cualquier maestro ajeno a la nueva Academia.

La conclusión parece razonable, pero dos cosas nos hacen dudar de ella. Para empezar, el carácter realista de la educación propuesta por el autor ${ }^{11}$ permite suponer en la nueva Academia la consciencia de que el contra omnia dicere es un método dialéctico para filósofos experimentados ${ }^{12}$. Por otro lado, ninguno de los jóvenes o aprendices que aparecen en los diálogos ciceronianos emprende una refutación contra los discursos que escuchan. El que asume el rol de maestro en esas obras únicamente pide a los iniciados sus juicios acerca de la verdad de los discursos. Opinión que, cuando se da, generalmente es muy escueta ${ }^{13}$. Es difícil suponer que se estimule en el iniciado la refutación de otras escuelas, pero no de los mismos maestros neoacadémicos.

11 Esto se puede percibir, por ejemplo, en su temor a la defatigatio en el proceso educativo.

12 El mismo Cicerón reconoce la dificultad del método, inmediatamente después del texto del De natura deorum analizado: "Cuius rei tantae tamque difficilis facultatem consecutum esse me non profiteor, secutum esse prae me fero" (12-13) IConfieso que no he alcanzado la habilidad de cosa tan importante y dificil, pero muestro haberlo intentado].

13 Lucullus (148); De finibus (II 119, V 76, V 96). El famoso final del De natura deorum (95). 
Quizá el contra omnia dicere haya estado presente desde los primeros momentos de la instrucción filosófica de dicha escuela; sin embargo, en tanto que instrumento para que el aprendiz conozca en solitario otras doctrinas, tal vez se reservase, cuanto mucho, a alumnos avanzados. De lo que no pareciera haber duda es sobre la excelencia del método para anteponer la ratio a cualquier forma de auctoritas.

Entonces, ¿de qué modo el juicio frágil del iniciado puede abarcar todos los sistemas? Diálogos como el De oratore, el De finibus y el De natura deorum no son escritos para presentar la opinión de una escuela, sino todo lo que en el momento se discute entre las principales corrientes. Los susodichos desarrollan la exposición por medio del in utramque partem. De donde se puede conjeturar que es a través de este método dialéctico que el iniciado neoacadémico puede escuchar todo lo dicho (omnes res audire) por los demás sabios. No se trataría de que oiga las distintas doctrinas de boca de sus creadores, sino de la de sus maestros en la Academia. Con lo cual no se expondría su juicio frágil al prestigio de eruditos de otras escuelas. Quedaría en la responsabilidad del maestro neoacadémico estructurar discursos antagónicos, de tal manera que su auctoritas no influya en el juicio del aprendiz. Todo esto es lo que intenta Cicerón.

\section{Unum y autoridad}

A simple vista, se podría suponer que pedirle al aprendiz escuchar todas las doctrinas tiene una finalidad meramente práctica, pues asi se evita que se circunscriba a la autoridad de un solo maestro. Sin embargo, el omnes res audire plasma, en el plano de la enseñanza, la doctrina ciceroniana de la unidad de todos los saberes, fundada, a su vez, en la unidad de todas las cosas, tal como se constatará de inmediato. En libro III del De oratore, Craso se opone a que se divida el discurso entre qué se debe decir (un ámbito del saber: la inventio) y cómo se debe decir (otro ámbito del saber: la elocutio). Para fundamentarse, recurre a unos antiguos (veteres) que habrian afirmado que:

... todo, lo de arriba y lo de abajo, es uno, (unum), y que está regido por un mismo dinamismo y armonia de la naturaleza, y que no hay ningún género de cosas (genus rerum) que, o pueda seguir existiendo (constare) si lo arrancan de los demás, o bien 
si de él se viera privado, el resto pueda conservar su fuerza y eternidad (20-21)14.

Obsérvese cómo el saber no debe separase en virtud de una ontología de la unidad de todas las cosas. Nótese, igualmente, la preocupación por mostrar, en el fragmento, las consecuencias negativas de no mantenerse el Unum. Estas, en lo que concierne a la separación de las ciencias, podrian ser: pérdida del existir propio (constare) y la fuerza (vis) de los estudios desvinculados entre sí. Pero también se podría añadir que la separación de los saberes ocasionaria la fractura de los respectivos ámbitos del ser de esos estudios, lo que traería las consecuencias señaladas.

El vínculo necesario que se quiere resaltar entre la unidad de los saberes y la unidad de lo real se patentiza con el ejemplo que de inmediato Crasos ofrece, el cual tendría como fin hacer más comprensible el Unum de los antiguos (veteres):

... es también verdadera aquella afirmación de Platón acerca de que toda la doctrina de estas artes humanas y liberales se encuentra contenida dentro de un vinculo que las une, asi pues, considerada la esencia (vis) y la razón de esto, de donde son las causas y los fines de todas las cosas, aparece un maravilloso concierto y armonía entre todas las doctrinas (21-22)15. Trad. del autor.

De nuevo, aquí se postula la unidad de los saberes, calificada de maravillosa, sustentada en la unidad de lo real.

Los estudiosos han reflexionado sobre el origen y la naturaleza de este Unum ciceroniano. Wilkins (1892, p. 13.) relaciona el primer fragmento con la doctrina eleática del Uno ${ }^{16}$, mientras que el segundo con el Epinomis (p. 14, 992 a), diálogo adjudicado a Platón, pero cuya autenticidad ha sido cuestionada desde hace tiempo. Dilorenzo, al observar que los Una presentados ${ }^{17}$ no son producto de razonamientos, los considera simples ejemplos para comunicar cierta intuición de Unum (1978, p. 251). El problema escapa a los alcances del presente trabajo, pero, al poner de manifiesto el fundamento ontológico de la unidad de los saberes, resalta la importancia central de esta última doctrina.

14 Trad. de José Javier Iso, pero con modificaciones nuestras.

15 La traducción es nuestra.

16 Remite a Platón (Sofista, 242 d) y al Lucullus (II 118).

17 Craso también ofrece, para hacer comprensible la doctrina de la unidad de los antiguos veteres, el Unum que constituirian los múltiples aspectos de la elocuencia (De oratore III 22-24). 
Ahora bien, desde tal perspectiva, ¿cuáles son las consecuencias de la auctoritas en la disputa y la enseñanza filosófica? Al iniciado dejarse llevar por la opinión del prestigioso maestro, o el sabio imponer su juicio, la autoridad rompe el concierto y la armonía de los saberes desde la escuela misma, lo que ocasiona, si se toman en cuenta las advertencias de Craso, aprendizajes sin existencia propia (constare) y sin fuerza (vis), además de una realidad escindida, fragmentada.

\section{Conclusiones}

Algunos investigadores actuales afirman que la nueva Academia careció de doctrina, pues se reducía a dialécticos que, en el mero afán de sostener su escepticismo, se contentaban con refutar otros sistemas (Schofield, 2002, p. 224). Sin embargo, si se parte del testimonio analizado de Cicerón, puede entreverse una concordancia entre el rechazo neoacadémico a la autoridad, los métodos dialécticos utilizados, su visión omniabarcante del saber y su probable fundamento ontológico; tal concordancia manifestaría el esfuerzo por sistematizar un pensamiento, al menos en el plano de la enseñanza filosófica. Enseñanza que tendría por fin el desarrollo de la libertad de juicio en el ser humano, la conformación del saber como un todo indisociable y el resguardo de la fuerza y armonía de la unidad de lo real. Tales objetivos se buscarian con la implementación, fundamentalmente, del método dialéctico denominado in utramque partem.

El artículo de Asmis (2001), sobre la educación epicúrea, y el nuestro, dedicado a la educación neoacadémica, manifiestan el esfuerzo de dichas escuelas por ofrecer una enseñanza innovadora, no solo en relación con otras filosofías, sino con la pedagogía tradicional. Es de esperarse, en consecuencia, un esfuerzo similar en el estoicismo. Semejante esfuerzo innovador puede facilitar mucho material útil a la educación de hoy en día, bien sea por los fundamentos o por los métodos. No sería mala idea investigar al respecto. 


\section{Referencias}

Adcock, F. G. (2000). Roman Political Ideas and Practice. Ann Arbor: University of Michigan Press.

Asmis, E. (2001). Basic Education in Epicureanism. L. Too (Ed.), Education in Greek and Roman Antiquity (pp. 209-239). Leiden: Brill.

Atkins, J. (2013). Cicero on Politics and the Limits of Reason. Cambridge: Cambridge University Press.

Balsdon, J. P. V. D. (1960). Auctoritas, Dignitas, Otium. The Classical Quarterly, 10(1), pp. 43-50.

Baraz, Y. (2012). A Written Republic: Cicero's Philosophical Politics. Princeton: Princeton University Press.

Bonner, S. (2012). Education in Ancient Rome, From the Elder Cato to the younger Pliny. New York: Routledge.

Bowman, A., y Woolf, G. (Eds.). (1994). Literacy and Power in the Ancient world. Cambridge: Cambridge University Press.

Bravo, F. (1990). Introducción a la filosofia de Platón. Caracas: Eduven.

Brittain, C. (2001). Philo of Larissa: The Last of the Academic Sceptics. Oxford: Oxford University Press, 2001.

Brunt, P. A. (1988). The Fall of the Roman Republic. Oxford: Oxford University Press.

César. (1998). Guerra civil. (Trad. J. Ruiz). Madrid: Gredos.

Cicero. (1947). Orationes. (Ed. A. Clark). Oxford: Oxford University Press. 
Cicero. (1959). De senectute. De amicitia. De divinatione. (Trad. W. A. Falconer). Cambridge: Harvard University Press.

Cicero. (1967). Denatura deorum, Academica. (Trad. H. Rackham). London: Heinemann.

Cicerón. (1990). Cuestiones Académicas. (Trad. J. Pimentel). México: UNAM.

Cicerón. (1999a). Sobre la naturaleza de los dioses. (Trad. Á. Escobar). Madrid: Gredos.

Cicerón. (1999b). Sobre la adivinación. Sobre el destino. El Timeo. (Trad. Á. Escobar). Madrid: Gredos.

Cicerón. (2002). Sobre el orador. (Trad. J. J. Iso). Madrid: Gredos.

Culpepper, S. (2010). Catullus, Cicero, and a Society of Patrons. The Generation of the Text. Cambridge: Cambridge University Press.

Dilorenzo, R. (1978). The Critique of Socrates in Cicero's De oratore: Ornatus and Nature of Wisdom. Philosophy and Rhetoric, 11(4), pp. 247-261.

Dugan, J. (2005). Making a New Man: Ciceronian Self-Fashioning in the Rhetorical Works. Oxford: Oxford University Press.

Fox, M. (2007). Cicero's Philosophy of History. Oxford: Oxford University Press.

Gruen, E. (1995). The Last Generation of the Roman Republic. Berkeley: University of California Press.

Marrou, H. (1948). Histoire de l'éducation dans l'antiquité. Paris: Seuil.

Lévy, C. (1992). Cicero Academicus: Recherchessurles Académiques etsur la philosophie cicéronienne. Roma: École Française de Rome.

Morgan, T. (1998). Literate Education in the Hellenistic and Roman Worlds. Cambridge: Cambridge University Press. 
Pernot, L. (1993). La Rhétorique de l'éloge dans le monde greco-romain. Paris: Collection des Études Augustiniennes.

Plutarco. (2007). Vidas paralelas VI. (Trad. Juan Guzmán). Madrid: Gredos.

Schofield, M. (1986). Cicero for and against Divination. The Journal of Roman Studies, (76), pp. 47-65.

Schofield, M. (2002). History of Hellenistic Philosophy. Cambridge: Cambridge University Press.

Schofield, M. (2008). Ciceronian dialogue. G. Simon (Ed.), The End of Dialogue in Antiquity (pp. 63-84). Cambridge: Cambridge University Press.

Too, L. (2001). Education in Greek and Roman Antiquity. Leiden: Brill.

Van der Blom, H. (2010). Cicero's Role Models. The Political Strategy of a Newcomer. Oxford: Oxford University Press.

Wilkins, A. S. (1892). De oratore libri tres. Oxford: Clarendon Press. 